\title{
$S, S$-Dimethyl dithiocarbonate: A Novel Carbonyl Dication Synthon in the Synthesis of Ketones
}

\author{
Chiar-Dy Chen, Jui-Wen Huang, Man-kit Leung*, Huei-hsu Li \\ Department of Chemistry, National Taiwan University, Taipei, Taiwan, R. O. C.
}

Received 11 February 1998; accepted 10 April 1998

\begin{abstract}
We report herein the use of DMDTC as an effective carbonyl dication equivalent in ketone synthesis. According to our strategy, we also successfully devised a synthetic pathway for $S$-methyl (trimethylsilyl)thioacetate which may be a potentially useful synthetic reagent in organic synthesis.

0 1998 Elsevier Science Ltd. All rights reserved.
\end{abstract}

Ketones are an important class of organic compounds whose synthesis and reactions have been actively studied during the last few decades. 1 In a retrosynthetic analysis for the formation of ketones, one can reasonably disconnect them into two carbanionic fragments as well as a carbonyl dication equivalent. Although this disconnection approach is simple and straightforward, known examples of this approach are rare. ${ }^{2}$ In addition, almost all the known carbonyl dication synthons in ketone synthesis are indeed prepared from phosgene. As our continuing efforts in developing mild reagents that can be used to substitute phosgene or its derivatives in organic synthesis, we recently discovered that $S, S$-dimethyl dithiocarbonate (DMDTC) is a very useful reagent in urea synthesis. ${ }^{3}$ With regard to the facts that DMDTC is structually similar to phosgene and could be prepared from methanol, carbon disulfide, and dimethyl sulfate through a two-step sequence, DMDTC is a potential candidate to substitute phosgene and its derivatives as an effective carbonyl dication synthon in ketone synthesis.

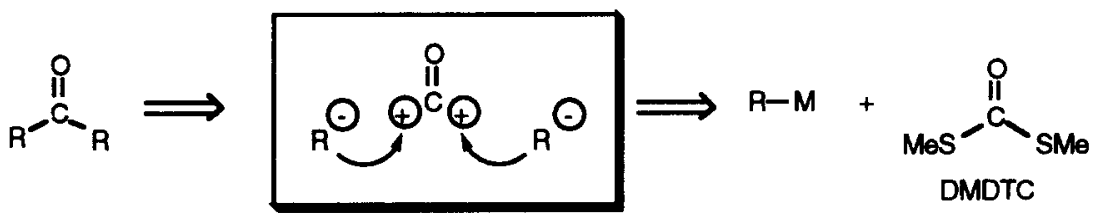

Our primary efforts are focused on developing an appropriate transition-metal catalyst that can effectively mediate the coupling reaction of DMDTC with Grignard or organolithium reagents. Since $\mathrm{Fe}(\mathrm{acac})_{3}$ and $\mathrm{CuI}$ are excellent promoters in the conversion of thioesters to ketones, $2 \mathrm{a}, 4$ they are potential candidates to investigate.

In an exploratory investigation carried out in the early stage of our study, we discovered that DMDTC does not react with hexylmagnesium bromide at $-78^{\circ} \mathrm{C}$. Gradually warming up the reaction mixture only lead to a complicated product mixture. In addition, our preliminary studies revealed that $\mathrm{Fe}(\mathrm{acac})_{3}$ is not an ideal catalyst. Reaction of hexylmagnesium bromide with DMDTC in the presence of catalytic amounts of $\mathrm{Fe}(\mathrm{acac})_{3}$ gives a product mixture of the corresponding thioester, ketone, and tertiary alcohol. Variation of the reaction 
temperatures or the amounts of $\mathrm{Fe}(\mathrm{acac})_{3}$ do not significantly improve the product ratios. However, dihexylmagnesiocuprate reacts with DMDTC to afford ketone 2 exclusively in high yield (Table 1). The potential efficiency of this reagent attracted our attention and prompted further investigation.

In a typical run for the formation of $2, \mathrm{CH}_{3}\left(\mathrm{CH}_{2}\right)_{5} \mathrm{MgBr}$ was treated with $\mathrm{CuI}$ at $-50{ }^{\circ} \mathrm{C}$ in THF under argon for 2 hours, followed by addition of a solution of DMDTC. The reaction mixture was kept at $-50^{\circ} \mathrm{C}$ for 0.5 hour and then warmed up gradually to room-temperature for 4 hours. After typical workup and purification procedures, ketone 2 was obtained in pure form. The success of this process is highly dependent upon the extent of complete conversion of hexylbromide to the corresponding Grignard reagent in the previous step. The presence of any residual hexylbromide would destroy the cuprate reagent during the course of its formation and therefore retard the desired reaction. This procedure works well for most of the unhindered primary and secondary alkylmagnesium halides in our trials (Table 1) except in some cases, modifications of the reaction conditions are needed.

Perhaps due to intramolecular complexation, ${ }^{5}$ aryl and alkoxy substituted Grignard reagents are less reactive than hexylmagnesium bromide towards cuprate formation. As a consequence, formation of these kinds of cuprates at $-50^{\circ} \mathrm{C}$ is sluggish and incomplete. The presence of the unreacted Grignard reagents would consume the newly formed ketones, resulting tertiary alcohols as the final products (Table 2). For reasons of steric hindrance, unreacted cyclopentylmagnesium bromide (5) acts as a reducing agent rather than a nucleophile, transferring a hydride to 6 to provide 21 in moderate yield. In order to improve the yields of the desired ketones, higher reaction temperatures are usually required (entries 3-10 in Table 1) to ensure the cuprate formations

Unlike other aryl substituted cuprates, reaction of dibenzylmagnesio cuprate with DMDTC only afforded alcohol 26 as the product. Increasing the cuprate-formation temperature would not improve the results, only giving significant amounts of dibenzyl as the side product. Nevertheless, we discovered that thioester formation could be mediated by $\mathrm{Ni}(\mathrm{acac})_{2}$ at $-78^{\circ} \mathrm{C}$ to give 27 in moderate yield.

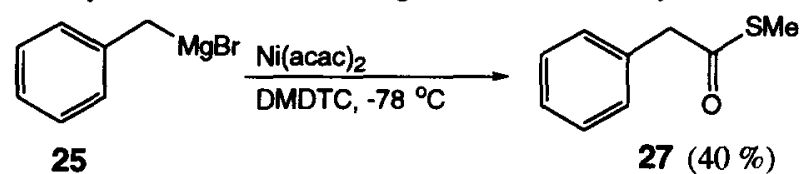

Although the reasons are not immediately obvious, ketone formation from 11 under typical reaction conditions is unsuccessful, giving a product mixture of $12 \mathbf{a}, \mathbf{1 2 b}$, and the corresponding tertiary alcohol. However. the reaction could be stopped at the stage of thioester formation by controlling the reaction temperature at $-50^{\circ} \mathrm{C}$ to give $12 \mathrm{a}$ as the major product. In addition, the complication in the formation of $\mathbf{1 2 b}$ could be subdued by employing one more equivalent of $\mathrm{CuI}$ in the reaction, affording $12 \mathrm{~b}$ in acceptable yield.

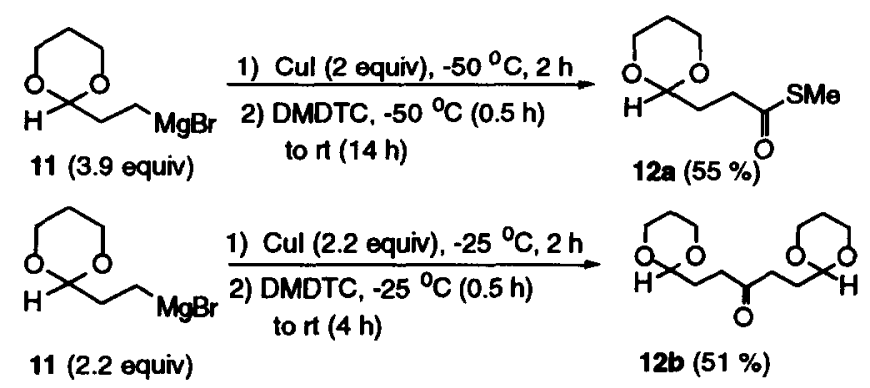


Table 1: Formation of Symmetrical Ketones by Reaction of DMDTC with various Cuprate

\section{Reagents}

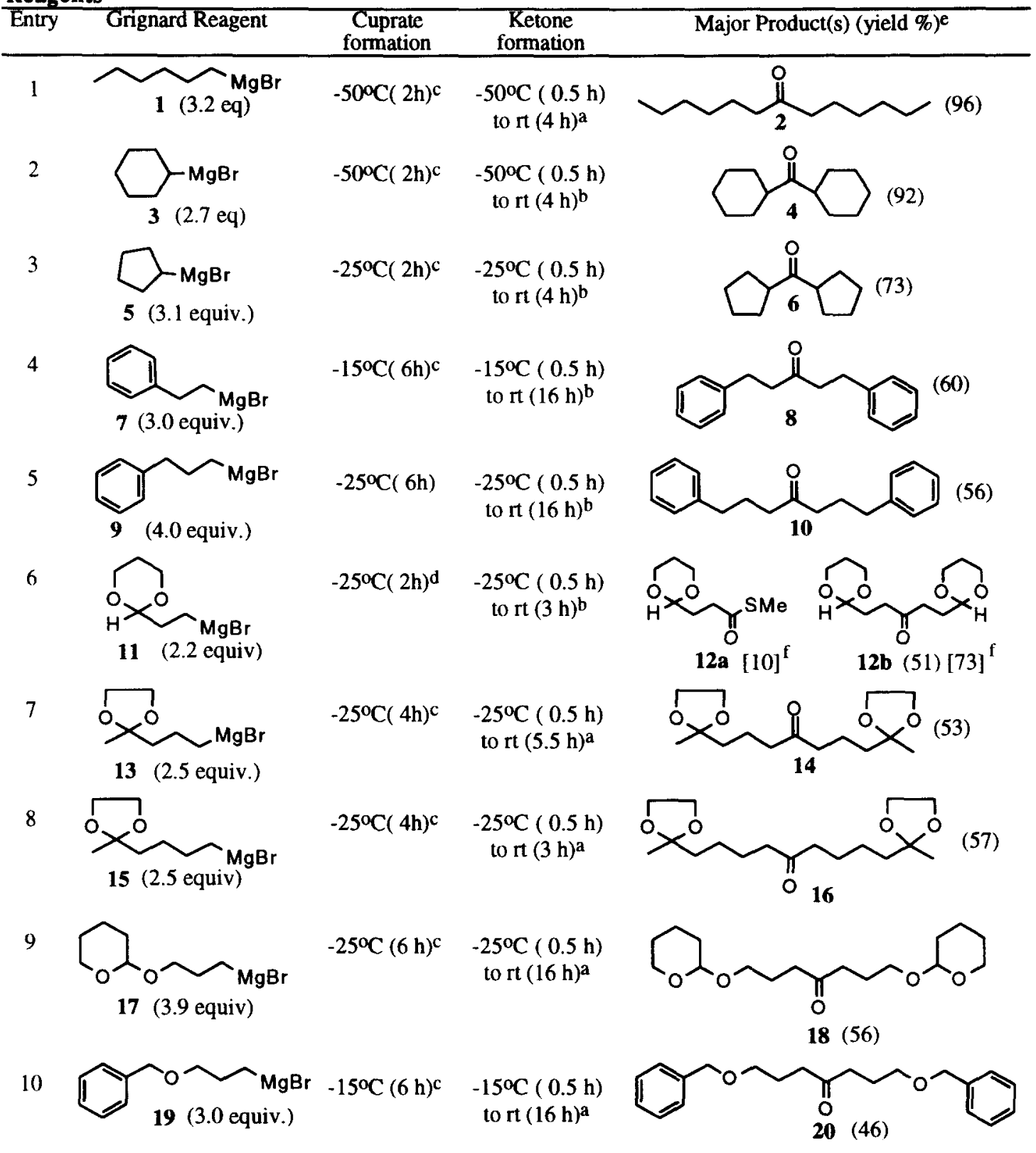

$a$, in THF; $b$, in ether; $c$, half an equivalent of CuI with respect to the Grignard reagent was used; $d$, one equivalent of $\mathrm{CuI}$ with respect to the Grignard reagent was used; e. isolated yield; f, GC yield. 
Table 2: CuI Mediated Reaction of DMDTC with Aryl or Alkoxy Substituted Grignard Reagents at $-50{ }^{\circ} \mathrm{C}$

$\stackrel{\substack{\mathrm{RMgBr} \\(3-3.5 \text { equiv.) }}}{\stackrel{\mathrm{Cul},-50^{\circ} \mathrm{C}}{2 \mathrm{~h}}} \stackrel{\frac{\mathrm{DMDTC}}{\longrightarrow}}{\stackrel{\begin{array}{l}-50^{\circ} \mathrm{C}, 0.5 \mathrm{~h} \text { and } \\ \text { room temperature, } 6-16 \mathrm{~h}\end{array}}{\longrightarrow}}$

Grignard Reagent<smiles>Br[Mg]C1CCCC1</smiles>

5<smiles>Br[Mg]CCc1ccccc1</smiles>

7<smiles>Br[Mg]C(Br)(Br)c1ccccc1</smiles>

9<smiles>Br[Mg]C(Br)(Br)c1ccccc1</smiles>

19<smiles>Cl[Mg]Cc1ccccc1</smiles>

25 Major Product(s) (yield \%)<smiles>OC(C1CCCC1)C1CCCC1</smiles>
21 (52\%)<smiles>CC(C)(O)CCc1ccccc1</smiles>
22 (41\%)<smiles>CC(C)(O)CCCc1ccccc1</smiles>

23 (34\%)

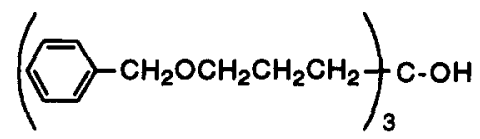

24 (47 \%)<smiles>CC(C)(O)Cc1ccccc1</smiles>

$26(53 \%)$

a. half an equivalent of $C u I$ with respect to the Grignard reagents was used; $b$, isolated yield with respect to the amount of DMDTC.

It is worth mentioning that formation of ketone $\mathbf{3 0}$ from sterically hindered Grignard reagent $\mathbf{2 8}$ is ineffective, giving thioester 29 as the major product. By taking the advantages of this selectivity, we could efficiently synthesize $S$-methyl (trimethylsilyl)thioacetate 32 in one step. Thioester $\mathbf{3 2}$ is a potentially useful synthetic reagent whose higher $S$-alkyl or aryl homologs and oxo-analogue 33 proved to be a useful reagent in $\alpha, \beta$-unsaturated thioester synthesis, ${ }^{6}$ silylation, ${ }^{7}$ nucleophilic addition, ${ }^{8}$ as well as 1,1 -disubstituted ethylene formation. 9<smiles>CC(C)(C)CN[13Br]</smiles>

28 1) $\mathrm{Cul}_{,}-50^{\circ} \mathrm{C}(2 \mathrm{~h})$
2) $\mathrm{DMDTC},-50^{\circ} \mathrm{C}(0.5 \mathrm{~h})$
to it (3h)<smiles>CC(=O)CC(C)(C)C</smiles>

$29(42 \%)$<smiles>CC(C)(C)CC(=O)CC(C)(C)C</smiles>

$30(<1 \%)$<smiles>C[Si](C)(C)C[14Cl]</smiles>

31 (2.6 equiv.)<smiles>CS(C)(C)C(=O)O</smiles>
(DMDTC)<smiles>C[Si](C)(C)CC(=O)O[Na]</smiles>

$32(68 \%)$<smiles>CCOC(=O)C[Si](C)(C)C</smiles>

33

An oxo-analogue of 32 
In an effort to develop a general synthetic route for unsymmetrical ketones, we examined the reaction of DMDTC with less reactive organocopper reagents. After exploring a number of reaction conditions, we eventually found that reaction of $\mathrm{RCu}-\mathrm{PPh}_{3}$ with DMDTC would result $\mathrm{R}(\mathrm{C}=\mathrm{O}) \mathrm{SMe}$ selectively in moderate yields. Due to the low reactivity of the organocopper reagents, significant amounts of the starting DMDTC were recovered after reaction.

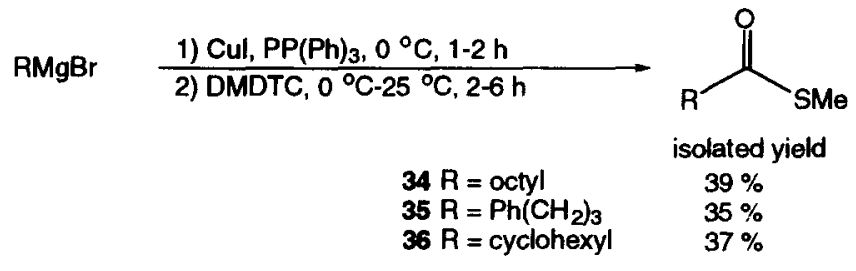

Our experiments first demonstrate that DMDTC can be an effective carbonyl dication equivalent in ketone synthesis. Oxygen-containing functional groups such as acetals and ethers can be tolerated. On the basis of our strategy, we could prepare 32 efficiently in good yield. The applications of 32 are under investigation.

\section{ACKNOWLEDGMENT}

We thank the National Science Council of the Republic of China for the financial support (NSC 862113-M-002-027 and NSC 86-2113-M-002-033)

\section{EXPERIMENTAL}

General. THF and diethyl ether were distilled from sodium metal under $\mathrm{N}_{2}$ with benzophenone as indicator. All the reactions were carried out under a $\mathrm{N}_{2}$ or Ar atmosphere. DMDTC was prepared according to our recently published method. ${ }^{3}$ Reactions were typically monitored using either TLC on commercial silica gel plates or GC. IR spectra were recorded on a Bio-rad FTS 40 FT-IR spectrophotometer as neat film or KBr plate. ${ }^{1} \mathrm{H}$ NMR spectra were recorded on a Bruker AC300 (300 MHz) spectrometer. Chemical shifts $(\delta)$ are reported in ppm downfield from internal standard tetramethylsilane and coupling constants $(J)$ are reported in $\mathrm{Hertz}(\mathrm{Hz}) .{ }^{13} \mathrm{C}$ spectra were either obtained on a Bruker AC300 (75 MHz) spectrometer. Mass spectra were determined using a HP-5989A mass spectrometer. Mps. were measured on a Melt-temp II melting-point apparatus, Laboratory Devices, USA.

A Representative Procedure for the Formation of Symmetrical Ketones from DMDTC: Dihexyl Ketone (2). To a vigorously stirred suspension of $\mathrm{Mg}(0.58 \mathrm{~g}, 24 \mathrm{mmol})$ in THF (1 mL) was added catalytic amounts of $I_{2}$. A small portion of 1-bromohexane was added to initiate the reaction, followed by dropwise addition of a solution of 1-bromohexane (totally $3.3 \mathrm{~g}, 20 \mathrm{mmol}$ ) in $20 \mathrm{~mL}$ THF. After addition, the reaction mixture was further stirred for $30 \mathrm{~min}$. The concentration of the hexylmagnesium bromide $(1.07 \mathrm{M})$ was determined by titration, using $\mathrm{HCl}(1 \mathrm{~N})$ as standard and phenolphthalein as the indicator. The extent of conversion ( $92 \%$ ) was determined by GC analysis. 
To prepare dihexyl ketone (2), the hexylmagnesium bromide $(1.1 \mathrm{M}, 3.6 \mathrm{~mL}, 3.9 \mathrm{mmol})$ was slowly added to a suspension of $\mathrm{CuI}(0.38 \mathrm{~g}, 2.0 \mathrm{mmol})$ in THF $(2 \mathrm{~mL})$ at $-50^{\circ} \mathrm{C}$. The mixture was kept at $-50^{\circ} \mathrm{C}$ for 2 $h$, followed by addition of a solution of DMDTC $(0.15 \mathrm{~g}, 1.2 \mathrm{mmol})$ in THF $(1 \mathrm{~mL})$. The reaction mixture was further reacted at $-50{ }^{\circ} \mathrm{C}$ for $0.5 \mathrm{~h}$ and gradually warmed to room temperature (rt) for $4 \mathrm{~h}$. The reaction was quenched by using saturated $\mathrm{NH}_{4} \mathrm{Cl}$ solution. The crude product was extracted with $\mathrm{CH}_{2} \mathrm{Cl}_{2}$, washed with saturated $\mathrm{NaCl}$ solution, concentrated and purified through liquid chromatography on silica gel, using a solvent gradient from hexanes to $\mathrm{CH}_{2} \mathrm{Cl}_{2}$ /hexanes (2:1) as eluent, to afford essentially pure 2 as colorless crystals $(0.23$

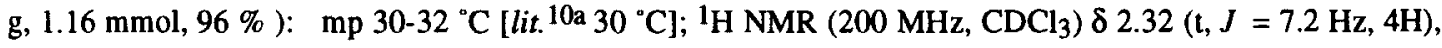
$1.53-1.42(\mathrm{~m}, 4 \mathrm{H}), 1.21(\mathrm{~m}, 12 \mathrm{H}), 0.84-0.78(\mathrm{t}, J=6.7 \mathrm{~Hz}, 6 \mathrm{H})\left[\right.$ lit. ${ }^{10 \mathrm{~b}}{ }^{\mathrm{l}} \mathrm{H}$ NMR $\delta 2.28(\mathrm{t}, 4 \mathrm{H}), 1.00-2.10$ $(\mathrm{m}, 16 \mathrm{H}), 0.70-1.00(\mathrm{~m}, 6 \mathrm{H})] ;{ }^{13} \mathrm{C}$ NMR $\left(75 \mathrm{MHz}, \mathrm{CDCl}_{3}\right) \delta 211.5,42.7,31.5,28.9,23.8,22.4,13.9$ [lit. $X X{ }^{13} \mathrm{C}$ NMR 210.8, 42.3, 31.2, 28.5, 23.4, 22.1, 13.5]; IR v $1713 \mathrm{~cm}^{-1}(\mathrm{C}=\mathrm{O}) ; \mathrm{MS} \mathrm{m} / \mathrm{z}$ (rel. intensity, EI, $20 \mathrm{eV}) 199\left(\mathrm{M}^{+}+1,35\right), 198\left(\mathrm{M}^{+}, 100\right), 169(5), 155(5), 141$ (10), $128(15), 113(30), 85$ (8); HRMS (70 $\mathrm{eV}, \mathrm{M}^{+}$) calcd. for $\mathrm{C}_{13} \mathrm{H}_{26} \mathrm{O}: 198.1985$, found 198.1982 .

Dicyclohexyl Ketone (4). The cyclohexylmagnesium bromide solution ( $0.87 \mathrm{M})$ was prepared from bromocyclohexane $(4.0 \mathrm{~g}, 25 \mathrm{mmol})$ and $\mathrm{Mg}(0.72 \mathrm{~g}, 30 \mathrm{mmol})$ in $\mathrm{Et}_{2} \mathrm{O}(25 \mathrm{~mL})$ according to the representative procedure. The dicyclohexyl ketone (4) was obtained by reaction of DMDTC $(0.31 \mathrm{~g}, 2.5 \mathrm{mmol})$ in $\mathrm{Et}_{2} \mathrm{O}(2.5$ $\mathrm{mL})$ with a mixture of $\mathrm{CuI}(0.64 \mathrm{~g}, 3.4 \mathrm{mmol})$ and cyclohexylmagnesium bromide $(0.87 \mathrm{M}, 7.7 \mathrm{~mL}, 6.7$ $\mathrm{mmol}$ ) at $-50{ }^{\circ} \mathrm{C}$ for $2 \mathrm{~h}$ and at $\mathrm{rt}$ for $4 \mathrm{~h}$. The reaction was worked up as usual and the crude product was purified through liquid chromatography on silica gel, using a solvent gradient from hexanes to $\mathrm{CH}_{2} \mathrm{Cl}_{2} /$ hexanes (2:1) as eluent, to afford essentially pure 4 as colorless crystals $(0.45 \mathrm{~g}, 2.3 \mathrm{mmol}, 92 \%)$ : ${ }^{1} \mathrm{H}$ NMR (300 $\left.\mathrm{MHz}, \mathrm{CDCl}_{3}\right) \delta 2.46-2.36(\mathrm{~m}, 2 \mathrm{H}), 1.73-1.62(\mathrm{~m}, 10 \mathrm{H}), 1.36-1.14(\mathrm{~m}, 10 \mathrm{H})\left[\right.$ [it. ${ }^{11 \mathrm{a}}{ }^{1_{\mathrm{H}}} \mathrm{NMR} \delta 2.20-2.60$ (t, 2H), 1.00-2.00 (m, 20 H)]; ${ }^{13} \mathrm{C} \mathrm{NMR}\left(75 \mathrm{MHz}, \mathrm{CDCl}_{3}\right) \delta 217.0,49.1,28.5,25.8,25.7\left[\right.$ lit. ${ }^{1{ }^{11}}{ }^{13} \mathrm{C}$ NMR $\delta$ 216.4, 48.9, 28.4, 25.7, 25.5]; IR v $1704 \mathrm{~cm}^{-1}(\mathrm{C}=\mathrm{O})$; MS m/z (rel. intensity, EI, $\left.20 \mathrm{eV}\right) 194\left(\mathrm{M}^{+}, 15\right), 111$ (50), 83 (100); HRMS ( $70 \mathrm{eV}, \mathrm{M}^{+}+1$ ) calcd. for $\mathrm{C}_{13} \mathrm{H}_{23} \mathrm{O}$ : 195.1750 , found 195.1750 .

Dicyclopentyl Ketone (6). The cyclopentylmagnesium bromide solution ( $0.82 \mathrm{M})$ was prepared from 1bromocyclopentane $(7.5 \mathrm{~g}, 50 \mathrm{mmol})$ and $\mathrm{Mg}(1.2 \mathrm{~g}, 50 \mathrm{mmol})$ in $\mathrm{Et}_{2} \mathrm{O}(50 \mathrm{~mL})$ according to the representative procedure. The corresponding cuprate reagent was prepared from $\mathrm{CuI}(1.6 \mathrm{~g}, 8.2 \mathrm{mmol})$ and cyclopentylmagnesium bromide $(0.82 \mathrm{M}, 20 \mathrm{~mL}, 16 \mathrm{mmol})$ at $-25{ }^{\circ} \mathrm{C}$ for $2 \mathrm{~h}$. To the cuprate solution, a solution of DMDTC $(0.62 \mathrm{~g}, 5.1 \mathrm{mmol})$ in $\mathrm{Et}_{2} \mathrm{O}(5 \mathrm{~mL})$ was added at $-25^{\circ} \mathrm{C}$. After reaction at $-25^{\circ} \mathrm{C}$ for $0.5 \mathrm{~h}$ and at $\mathrm{rt}$ for $4 \mathrm{~h}$, the mixture was worked up according to the representative procedure to provide a crude oil which was purified through liquid chromatography on silica gel, using a solvent gradient from hexanes to $\mathrm{CH}_{2} \mathrm{Cl}_{2}$ /hexane $=1 / 2$ as eluent to obtain $6(0.61 \mathrm{~g}, 3.7 \mathrm{mmol}, 72 \%)$ as a colorless solid: ${ }^{1} \mathrm{H} \mathrm{NMR}(300 \mathrm{MHz}$, $\left.\mathrm{CDCl}_{3}\right) \delta 2.95-2.79(\mathrm{~m}, 2 \mathrm{H}), 1.71-1.39(\mathrm{~m}, 16 \mathrm{H})\left[\right.$ lit. $\left.{ }^{12} \mathrm{l}_{\mathrm{H}} \mathrm{NMR} \delta 2.98(2 \mathrm{H}), 1.30-2.00(16 \mathrm{H})\right] ;{ }^{13} \mathrm{C}$ NMR $\left(75 \mathrm{MHz}, \mathrm{CDCl}_{3}\right): \delta 216.0,50.5,29.2,25.9$ [lit. ${ }^{11 \mathrm{~b}}{ }^{13} \mathrm{C}$ NMR $\left.\delta 215.0,50.0,28.6,25.5\right] ;$ IR $v 1706 \mathrm{~cm}^{-1}$ $(\mathrm{C}=0)$; MS m/z (rel. intensity, EI, $20 \mathrm{eV}) 166\left(\mathrm{M}^{+}, 10\right), 97(50), 69(100)$; HRMS (70 eV, $\left.\mathrm{M}^{+}\right)$calcd. for $\mathrm{C}_{11} \mathrm{H}_{18} \mathrm{O}$ : 166.1359 , found 166.1355 .

1,5-Diphenyl-3-pentanone (8). The 2-phenylethylmagnesium bromide solution $(0.87 \mathrm{M})$ was prepared from 2-bromoethylbenzene $(2.5 \mathrm{~g}, 14 \mathrm{mmol})$ and $\mathrm{Mg}(0.4 \mathrm{~g}, 17 \mathrm{mmol})$ in $\mathrm{Et}_{2} \mathrm{O}(15 \mathrm{~mL})$ according to the representative procedure. The corresponding cuprate reagent was prepared from $\mathrm{CuI}(0.83 \mathrm{~g}, 4.4 \mathrm{mmol})$ in $\mathrm{Et}_{2} \mathrm{O}$ 
$(4 \mathrm{~mL})$ and 2-phenylethylmagnesium bromide $(0.87 \mathrm{M}, 8 \mathrm{~mL}, 7.2 \mathrm{mmol})$ at $-15^{\circ} \mathrm{C}$ for $6 \mathrm{~h}$. To the cuprate solution, a solution of DMDTC $(0.27 \mathrm{~g}, 2.2 \mathrm{mmol})$ in $\mathrm{Et}_{2} \mathrm{O}(2 \mathrm{~mL})$ was added. After reaction at $-15^{\circ} \mathrm{C}$ for 0.5 $\mathrm{h}$ and at $\mathrm{rt}$ for $16 \mathrm{~h}$. the mixture was worked up according to the representative procedure to provide a crude oil which was purified through liquid chromatography on silica gel, using a solvent gradient from hexanes to $\mathrm{CH}_{2} \mathrm{Cl}_{2}$ /hexane $=1 / 2$ as eluent to obtain $8(0.31 \mathrm{~g}, 1.3 \mathrm{mmol}, 60 \%)$ as a colorless solid: ${ }^{1} \mathrm{H}$ NMR (300 $\left.\mathrm{MHz} \mathrm{CDCl}_{3}\right) \delta$ 7.30-7.25 (m, 4H), 7.21-7.14 (m, 6H), $2.89(\mathrm{t}, J=7.5 \mathrm{~Hz}, 4 \mathrm{H}), 2.70(\mathrm{t}, J=7.5 \mathrm{~Hz}, 4 \mathrm{H})$ $\left[\right.$ [it. ${ }^{13}{ }^{1} \mathrm{H}$ NMR $\left.\delta 7.20(2,10 \mathrm{H}), 2.70(\mathrm{~m}, 8 \mathrm{H})\right] ;{ }^{13} \mathrm{C}$ NMR $\left(75 \mathrm{MHz}, \mathrm{CDCl}_{3}\right) \delta 209.1,141.0,128.5,128.3$, 126.1, 44.5, 29.7; IR $\cup 1713 \mathrm{~cm}^{-1}(\mathrm{C}=\mathrm{O}) ; \mathrm{MS} \mathrm{m} / \mathrm{z}$ (rel. intensity, EI, $\left.20 \mathrm{eV}\right) 238\left(\mathrm{M}^{+}, 30\right), 220$ (5), $133(55)$, 105 (85), 91 (100), 77 (20): HRMS ( $\left.70 \mathrm{eV}, \mathrm{M}^{+}\right)$calcd. for $\mathrm{C}_{17} \mathrm{H}_{18} \mathrm{O}$ : 238.1359, found 238.1361.

1,7-Diphenyl-4-heptanone (10). The 3-phenylpropylmagnesium bromide solution (1.3 M) was prepared from 1-bromopropylbenzene $(6.0 \mathrm{~g}, 30 \mathrm{mmol})$ and $\mathrm{Mg}(0.79 \mathrm{~g}, 33 \mathrm{mmol})$ in $\mathrm{Et}_{2} \mathrm{O}(30 \mathrm{~mL})$ according to the representative procedure. The corresponding cuprate reagent was prepared from $\mathrm{CuI}(2.7 \mathrm{~g}, 14 \mathrm{mmol})$ and 3phenylpropylmagnesium bromide $(1.3 \mathrm{M}, 20 \mathrm{~mL}, 26 \mathrm{mmol})$ at $-25{ }^{\circ} \mathrm{C}$ for $6 \mathrm{~h}$. To the cuprate solution, a solution of DMDTC $(0.79 \mathrm{~g}, 6.5 \mathrm{mmol})$ in $\mathrm{Et}_{2} \mathrm{O}(6 \mathrm{~mL})$ was added at $-25^{\circ} \mathrm{C}$. After reaction at $-25^{\circ} \mathrm{C}$ for $0.5 \mathrm{~h}$ and at $\mathrm{rt}$ for $16 \mathrm{~h}$, the mixture was worked up according to the representative procedure to provide a crude oil which was purified through liquid chromatography on silica gel, using a solvent gradient from hexanes to $\mathrm{CH}_{2} \mathrm{Cl}_{2}$ /hexane $=1 / 2$ as eluent to obtain $10(0.97 \mathrm{~g}, 3.7 \mathrm{mmol}, 56 \%)$ as a colorless oil: ${ }^{1} \mathrm{H}$ NMR ( $300 \mathrm{MHz}$, $\left.\mathrm{CDCl}_{3}\right) \delta$ 7.35-7.30 (m, 4H), 7.25-7.19 (m, 6H), $2.65(\mathrm{t}, J=7.5 \mathrm{~Hz}, 4 \mathrm{H}), 2.41(\mathrm{t}, J=7.3 \mathrm{~Hz}, 4 \mathrm{H}), 1.99$ $1.89(\mathrm{~m}, 4 \mathrm{H}) ;{ }^{13} \mathrm{C}$ NMR $\left(75 \mathrm{MHz}, \mathrm{CDCl}_{3}\right) \delta 210.3,141.5,128.3,128.2,125.8,41.8,34.9,25.0$; IR $v$ $1711 \mathrm{~cm}^{-1}(\mathrm{C}=\mathrm{O}) ; \mathrm{MS} \mathrm{m} / \mathrm{z}$ (rel. intensity, EI, $\left.20 \mathrm{eV}\right) 266\left(\mathrm{M}^{+}, 8\right), 175(20), 162(8), 147$ (6), $104(100), 91$ (45); HRMS (70 eV, $\mathrm{M}^{+}$) calcd. for $\mathrm{C}_{19} \mathrm{H}_{22} \mathrm{O}: 266.1672$, found 266.1669 .

3-(1,3-Dioxane-2-yl)thiopropionic Acid S-Methyl Ester (12a). The 2-(1,3-Dioxane-2yl)ethylmagnesium bromide solution $(0.96 \mathrm{M})$ was prepared from 2-(2-bromoethyl)-1,3-dioxane $(2.0 \mathrm{~g}, 10$ $\mathrm{mmol})$ and $\mathrm{Mg}(0.25 \mathrm{~g}, 10.5 \mathrm{mmol})$ in THF $(10 \mathrm{~mL})$ according to the representative procedure. The corresponding cuprate reagent was prepared from $\mathrm{CuI}(0.81 \mathrm{~g}, 4.3 \mathrm{mmol})$ in THF $(4.5 \mathrm{~mL})$ and 2-(1,3-dioxane2-yl)ethylmagnesium bromide $(0.96 \mathrm{M}, 9.0 \mathrm{~mL}, 8.6 \mathrm{mmol})$ at $-50{ }^{\circ} \mathrm{C}$ for $2 \mathrm{~h}$. To the cuprate solution, a solution of DMDTC $(0.27 \mathrm{~g}, 2.2 \mathrm{mmol})$ in THF $(2.2 \mathrm{~mL})$ was added. After reaction at $-50{ }^{\circ} \mathrm{C}$ for $0.5 \mathrm{~h}$ and at $\mathrm{rt}$ for $14 \mathrm{~h}$, the mixture was worked up according to the representative procedure to provide a crude oil which was purified through liquid chromatography on silica gel, using a solvent gradient from hexanes $/ \mathrm{CH}_{2} \mathrm{Cl}_{2}=1 / 1$ to $1 / 2$ as eluent to obtain $12 \mathrm{a}(0.23 \mathrm{~g}, 1.2 \mathrm{mmol}, 55 \%)$ as a colorless oil: ${ }^{1} \mathrm{H}$ NMR $\left(300 \mathrm{MHz}, \mathrm{CDCl}_{3}\right) \delta 4.52$ $(\mathrm{t}, J=5.0 \mathrm{~Hz}, 1 \mathrm{H}), 4.03(\mathrm{dd}, J=12 \mathrm{~Hz}, 5 \mathrm{~Hz}, 2 \mathrm{H}), 3.70(\mathrm{dt}, J=12 \mathrm{~Hz}, 2.2 \mathrm{~Hz}, 2 \mathrm{H}), 2.64(\mathrm{t}, J=8.1 \mathrm{~Hz}$, $2 \mathrm{H}), 2.24(\mathrm{~s}, 3 \mathrm{H}), 2.04-1.96(\mathrm{~m}, 1 \mathrm{H}) \quad 1.93-1.86(\mathrm{~m}, 2 \mathrm{H}), 1.30-1.26(\mathrm{~d}, J=12 \mathrm{~Hz}, 1 \mathrm{H}) ;{ }^{13} \mathrm{C}$ NMR $(75$ $\left.\mathrm{MHz}_{1} \mathrm{CDCl}_{3}\right) \delta 199.3,100.5,66.7,37.9,30.4,25.6,11.5$; IR (neat) $v 1692 \mathrm{~cm}^{-1}(\mathrm{C}=\mathrm{O}) ; \mathrm{MS} \mathrm{m} / \mathrm{z}$ (rel. intensity, EI, $20 \mathrm{eV}) 189\left(\mathrm{M}^{+}-1,10\right), 175$ (13), 143 (100), 85 (95), 100 (20); HRMS (70 eV, $\mathrm{M}^{+}$) calcd. for $\mathrm{C}_{8} \mathrm{H}_{14} \mathrm{O}_{3} \mathrm{~S}: 190.0664$, found 190.0662 .

1,5-Bis(1,3-dioxane-2-yl)-3-pentanone (12b). The Grignard solution $(0.96 \mathrm{M})$ was prepared according to the previous procedure. The corresponding cuprate reagent was prepared from $\mathrm{CuI}(0.98 \mathrm{~g}, 4.7$ $\mathrm{mmol})$ in THF ( $4 \mathrm{~mL}$ ) and 2-(1,3-dioxane-2-yl)ethylmagnesium bromide $(0.96 \mathrm{M}, 5.0 \mathrm{~mL}, 4.7 \mathrm{mmol})$ at $-25^{\circ} \mathrm{C}$ for $4 \mathrm{~h}$. To the cuprate solution, a solution of DMDTC $(0.25 \mathrm{~g}, 2.0 \mathrm{mmol})$ in THF $(2.0 \mathrm{~mL})$ was added. After 
reaction at $-25{ }^{\circ} \mathrm{C}$ for $0.5 \mathrm{~h}$ and at $\mathrm{nt}$ for $15 \mathrm{~h}$, the mixture was worked up according to the representative procedure to provide a crude oil which was purified through liquid chromatography on silica gel, using hexanes/ethyl acetate $=1 / 3$ as eluent to obtain $12 \mathrm{~b}(0.26 \mathrm{~g}, 1.0 \mathrm{mmol}, 51 \%)$ as a colorless oil: ${ }^{1} \mathrm{H}$ NMR $\left(300 \mathrm{MHz}, \mathrm{CDCl}_{3}\right) \delta 4.47(\mathrm{t}, J=5.0 \mathrm{~Hz}, 2 \mathrm{H}), 4.01-2.96(\mathrm{dd}, J=12 \mathrm{~Hz}, 5 \mathrm{~Hz}, 4 \mathrm{H}), 3.65(\mathrm{dt}, J=12 \mathrm{~Hz}, 3.3$ $\mathrm{Hz}, 4 \mathrm{H}), 2.45(\mathrm{t}, J=7.2 \mathrm{~Hz}, 4 \mathrm{H}), 2.04-1.96(\mathrm{~m}, 2 \mathrm{H}), 1.78(\mathrm{dt}, J=7.2 \mathrm{~Hz}, 5 \mathrm{~Hz}, 4 \mathrm{H}), 1.27-1.22(\mathrm{~d}, J=$ $12.3 \mathrm{~Hz}, 2 \mathrm{H}$ ); ${ }^{13} \mathrm{C}$ NMR $\left(75 \mathrm{MHz}, \mathrm{CDCl}_{3}\right) \delta 209.6,100.8,66.7,36.5,28.9,25.6$; IR v $1714 \mathrm{~cm}^{-1}(\mathrm{C}=0)$; MS m/z (rel. intensity, EI, $20 \mathrm{eV}) 258\left(\mathrm{M}^{+}, 5\right), 257\left(\mathrm{M}^{+}-1,10\right), 156$ (23), 143 (24), 100 (100), 87 (60); HRMS ( $70 \mathrm{eV}, \mathrm{M}^{+}-1$ ) calcd. for $\mathrm{C}_{12} \mathrm{H}_{21} \mathrm{O}_{4}: 258.1468$, found 258.1458 .

1,7-Bis(2-methyl-1,3-dioxolane-2-yl)-4-heptanone (14). To a vigorously stirred mixture of $\mathrm{Mg}$ $(0.60 \mathrm{~g}, 25 \mathrm{mmol})$ and 2-(3-chloropropyl)-2-methyl-1,3-dioxolane $(2.0 \mathrm{~g}, 12 \mathrm{mmol})$ in THF $(15 \mathrm{~mL})$ was added catalytic amounts of $\mathrm{I}_{2}$ and two drops of dibromoethane. The mixture was warmed at $80{ }^{\circ} \mathrm{C}$ and two drops of methylmagnesium bromide $(1.0 \mathrm{M})$ was added to initiate the reaction. After addition, the reaction mixture was kept at reflux for $2 \mathrm{~h}$ to obtain 3-(2-methyl-1,3-dioxolane-2-yl)propylmagnesium chloride (0.90 M). The corresponding cuprate reagent was prepared from CuI $(1.0 \mathrm{~g}, 5.4 \mathrm{mmol})$ in THF $(5 \mathrm{~mL})$ and 3-(2methyl-1,3-dioxolane-2-yl)propylmagnesium chloride $(0.90 \mathrm{M}, 12.0 \mathrm{~mL}, 10.8 \mathrm{mmol})$ at $-25^{\circ} \mathrm{C}$ for $4 \mathrm{~h}$. To the cuprate solution, a solution of DMDTC $(0.54 \mathrm{~g}, 4.4 \mathrm{mmol})$ in THF $(4.0 \mathrm{~mL})$ was added. After reaction at -25 ${ }^{\circ} \mathrm{C}$ for $0.5 \mathrm{~h}$ and at $\mathrm{rt}$ for $5.5 \mathrm{~h}$, the mixture was worked up according to the representative procedure to provide a crude oil which was purified through liquid chromatography on silica gel, using a gradient eluent from hexanes/ethyl acetate $=1 / 6$ to hexanes/ethyl acetate $=1 / 3$ to obtain $14(0.66 \mathrm{~g}, 2.31 \mathrm{mmol}, 53 \%)$ as a colorless oil: ${ }^{1} \mathrm{H}$ NMR (300 MHz, CD $\left.{ }_{3} \mathrm{CN}\right) \delta 3.79-3.75(\mathrm{~m}, 8 \mathrm{H}), 2.27(\mathrm{t}, J=6.8 \mathrm{~Hz}, 4 \mathrm{H}), 1.55-1.44(\mathrm{~m}, 8 \mathrm{H}), 1.15$ (s, 6H): ${ }^{13} \mathrm{C}$ NMR (75 MHz, CD $\left.3 \mathrm{CN}\right) \delta 210.2,109.5,64.3,42.2,38.0,23.4,18.0$; IR $v 1711 \mathrm{~cm}^{-1}(\mathrm{C}=\mathrm{O})$; MS m/z (rel. intensity, EI, $20 \mathrm{eV}) 287\left(\mathrm{M}^{+}+1,20\right), 271(8), 99(20), 87(100)$; HRMS $\left(70 \mathrm{eV}, \mathrm{M}^{+}\right)$calcd. for $\mathrm{C}_{15} \mathrm{H}_{26} \mathrm{O}_{5}: 286.1781$, found 286.1788 .

1,9-Bis(2-methyl-1,3-dioxolane-2-yl)-5-nonanone (16) To a vigorously stirred mixture of $\mathrm{Mg}(0.80$ $\mathrm{g}, 33 \mathrm{mmol}$ ) and 2-(4-chlorobutyl)-2-methyl-1,3-dioxolane (1.8 g, $9.9 \mathrm{mmol})$ in THF (13 mL) was added catalytic amounts of $I_{2}$ and four drops of dibromoethane. After addition, the reaction mixture was kept at reflux for $20 \mathrm{~h}$ to obtain 4-(2-methyl-1,3-dioxolane-2-yl)butylmagnesium chloride $(0.90 \mathrm{M})$. The corresponding cuprate reagent was prepared from $\mathrm{CuI}(0.84 \mathrm{~g}, 4.4 \mathrm{mmol})$ in THF $(4 \mathrm{~mL})$ and 4-(2-methyl-1,3-dioxolane-2yl)butylmagnesium chloride $(0.90 \mathrm{M}, 1.0 \mathrm{~mL}, 8.8 \mathrm{mmol})$ at $-25^{\circ} \mathrm{C}$ for $4 \mathrm{~h}$. To the cuprate solution, a solution of DMDTC $(0.43 \mathrm{~g}, 3.5 \mathrm{mmol})$ in THF $(3.0 \mathrm{~mL})$ was added. After reaction at $-25{ }^{\circ} \mathrm{C}$ for $0.5 \mathrm{~h}$ and at $\mathrm{rt}$ for $3 \mathrm{~h}$, the mixture was worked up according to the representative procedure to provide a crude oil which was purified through liquid chromatography on silica gel, using a gradient eluent from hexanes/ethyl acetate $=1 / 7$ to hexanes/ethyl acetate $=1 / 3$ to obtain $16(0.62 \mathrm{~g}, 2.0 \mathrm{mmol}, 57 \%)$ as a colorless oil: ${ }^{1} \mathrm{H}$ NMR (300 MHz, $\left.\mathrm{CD}_{3} \mathrm{CN}\right) \delta 3.92-3.82(\mathrm{~m}, 8 \mathrm{H}), 2.41(\mathrm{t}, J=7.2 \mathrm{~Hz}, 4 \mathrm{H}), 1.63-1.46(\mathrm{~m}, 8 \mathrm{H}), 1.39-1.28(\mathrm{~m}, 4 \mathrm{H}), 1.25(\mathrm{~s}, 6 \mathrm{H})$; ${ }^{13} \mathrm{C}$ NMR $\left(75 \mathrm{MHz}, \mathrm{CD}_{3} \mathrm{CN}\right) \delta 212.1,110.8,65.6,43.4,40.0,25.1,24.8,24.4$; IR $v 1711 \mathrm{~cm}^{-1}(\mathrm{C}=\mathrm{O})$; MS $\mathrm{m} / \mathrm{z}$ (rel. intensity, EI, $20 \mathrm{eV}) 314\left(\mathrm{M}^{+}, 0.6\right), 299\left(\mathrm{M}^{+}-15,12\right), 128(6), 87(100)$; HRMS (70 eV, $\left.\mathbf{M}^{+}\right)$calcd. for $\mathrm{C}_{17} \mathrm{H}_{30} \mathrm{O}_{5}$ : 314.2095, found 314.2101.

1,7-Bis(tetrahydropyran-2-yloxy)-4-heptanone (18). The 3-(tetrahydropyran-2-yloxy)propylmagnesium bromide solution $(0.88 \mathrm{M})$ was prepared from 2-(3-bromopropoxy)tetrahydrofuran $(3.0 \mathrm{~g}, 14$ 
$\mathrm{mmol})$ and $\mathrm{Mg}(0.97 \mathrm{~g}, 40 \mathrm{mmol})$ in THF (13 mL) according to the representative procedure. The corresponding cuprate reagent was prepared from CuI $(1.1 \mathrm{~g}, 5.9 \mathrm{mmol})$ in THF $(5 \mathrm{~mL})$ and 3(tetrahydorpyran-2-yloxy)propylmagnesium bromide $(0.88 \mathrm{M}, 13.0 \mathrm{~mL}, 11 \mathrm{mmol})$ at $-25{ }^{\circ} \mathrm{C}$ for $6 \mathrm{~h}$. To the cuprate solution, a solution of DMDTC $(0.36 \mathrm{~g}, 2.9 \mathrm{mmol})$ in THF $(2.0 \mathrm{~mL})$ was added. After reaction at - 25 ${ }^{\mathrm{C}} \mathrm{C}$ for $0.5 \mathrm{~h}$ and at $\mathrm{rt}$ for $16 \mathrm{~h}$, the mixture was worked up according to the representative procedure to provide a crude oil which was purified through liquid chromatography on silica gel, using hexanes/ethyl acetate $=1 / 2$ as eluent to obtain $18(0.51 \mathrm{~g}, 1.6 \mathrm{mmol}, 56 \%)$ as a colorless oil: ${ }^{1} \mathrm{H}$ NMR $\left(300 \mathrm{MHz}, \mathrm{CDCl}_{3}\right) \delta 4.45(\mathrm{t}, J=$ $3.3 \mathrm{~Hz}, 2 \mathrm{H}), 3.74-3.59(\mathrm{~m}, 4 \mathrm{H}), 3.41-3.29(\mathrm{~m}, 4 \mathrm{H}), 2.45(\mathrm{t}, J=7.1 \mathrm{~Hz}, 4 \mathrm{H}), 1.82-1.42(\mathrm{~m}, 16 \mathrm{H}) ;{ }^{13} \mathrm{C}$ NMR $\left(75 \mathrm{MHz}, \mathrm{CDCl}_{3}\right) \delta 210.2,98.7,66.4,62.2,39.4,30.5,25.3,23.8,19.5 ; \mathrm{IR} \cup 1713 \mathrm{~cm}^{-1}(\mathrm{C}=0)$; MS $\mathrm{m} / \mathrm{z}$ (rel. intensity, EI, $20 \mathrm{eV}) 315\left(\mathrm{M}^{+}+1,0.1\right), 314\left(\mathrm{M}^{+}, 0.09\right), 213\left(\mathrm{M}^{+}-101,7\right), 129(50), 111(10), 85$ (100); HRMS ( $70 \mathrm{eV}, \mathrm{M}^{+}$) calcd. for $\mathrm{C}_{17} \mathrm{H}_{30} \mathrm{O}_{5}$ : 314.2095, found 314.2087 .

1,7-Bis(benzyloxy)heptan-4-one (20). The 3-(benzyloxy)propylmagnesium bromide solution (0.90 M) was prepared from 1-benzyloxy-3-bromopropane $(2.0 \mathrm{~g}, 8.7 \mathrm{mmol})$ and $\mathrm{Mg}(0.42 \mathrm{~g}, 18 \mathrm{mmol})$ in THF (8.5 $\mathrm{mL})$ according to the representative procedure. The corresponding cuprate reagent was prepared from $\mathrm{CuI}(0.70$ g. $3.7 \mathrm{mmol}$ ) in THF ( $3 \mathrm{~mL}$ ) and 3-(benzyloxy)propylmagnesium bromide $(0.97 \mathrm{M}, 7.5 \mathrm{~mL}, 7.3 \mathrm{mmol})$. To the cuprate solution, a solution of DMDTC $(0.30 \mathrm{~g}, 2.5 \mathrm{mmol})$ in THF $(2.0 \mathrm{~mL})$ was added. After reaction at $-15^{\circ} \mathrm{C}$ for $6 \mathrm{~h}$ and at $\mathrm{rt}$ for $16 \mathrm{~h}$, the mixture was worked up according to the representative procedure to provide a crude oil which was purified through liquid chromatography on silica gel, using hexanes/ethyl acetate $=1 / 2$ as eluent to obtain $20(0.37 \mathrm{~g}, 1.1 \mathrm{mmol}, 46 \%)$ as a colorless oil: ${ }^{1} \mathrm{H}$ NMR $\left(300 \mathrm{MHz}, \mathrm{CDCl}_{3}\right) \delta$ 7.37-7.25 (m, $10 \mathrm{H}), 4.47(\mathrm{~s}, 4 \mathrm{H}), 3.47(\mathrm{t}, J=6.2 \mathrm{~Hz}, 4 \mathrm{H}), 2.52(\mathrm{t}, J=7.2 \mathrm{~Hz}, 4 \mathrm{H}), 1.93-1.84(\mathrm{~m}, 4 \mathrm{H}) ;{ }^{13} \mathrm{C}$ NMR $(75$ $\left.\mathrm{MHz}, \mathrm{CDCl}_{3}\right) \delta 210.1,138.3,128.1,127.4,127.3,72.6,69.1,39.2,23.7 ; \mathrm{IR}$ v $1713 \mathrm{~cm}^{-1}(\mathrm{C}=\mathrm{O}) ; \mathrm{MS} \mathrm{m} / \mathrm{z}$ (rel. intensity, EI. $20 \mathrm{eV}) 327\left(\mathrm{M}^{+}+1,10\right), 219$ (15), 129 (95), 111 (42), 106 (13), 91 (100); HRMS (70 eV, $\mathrm{M}^{+}$) calcd. for $\mathrm{C}_{21} \mathrm{H}_{26} \mathrm{O}_{3}: 326.1883$, found 326.1880 .

Di(cyclopentyl)methanol (21) To a suspension of $\mathrm{CuI}(1.6 \mathrm{~g}, 8.2 \mathrm{mmol})$ in $\mathrm{Et}_{2} \mathrm{O}(8 \mathrm{~mL})$ was added cyclopentylmagnesium bromide $(0.82 \mathrm{M}, 20 \mathrm{~mL}, 16 \mathrm{mmol})$ at $-50^{\circ} \mathrm{C}$. After addition, the mixture was stirred at $-50{ }^{\circ} \mathrm{C}$ for $2 \mathrm{~h}$ and a solution of DMDTC $(0.62 \mathrm{~g}, 5.1 \mathrm{mmol})$ in $\mathrm{Et}_{2} \mathrm{O}(5 \mathrm{~mL})$ was added. The mixture was further reacted at $-50^{\circ} \mathrm{C}$ for $6 \mathrm{~h}$ and quenched by addition of saturated $\mathrm{NH}_{4} \mathrm{Cl}$ solution. The crude product was extracted with $\mathrm{CH}_{2} \mathrm{Cl}_{2}$, washed with brine, dried over anhydrous $\mathrm{MgSO}_{4}$, concentrated under reduced pressure, and purified through liquid chromatography on silica gel, using a solvent gradient from hexanes to $\mathrm{CH}_{2} \mathrm{Cl}_{2}$ /hexanes $=1 / 1$ as eluent to obtain 21 as colorless crystals $(0.45 \mathrm{~g}, 2.7 \mathrm{mmol}, 52 \%): \mathrm{mp} 46-48{ }^{\circ} \mathrm{C}$ [ lit. $^{14} 45^{\circ} \mathrm{C}$ ]; ${ }^{1} \mathrm{H}$ NMR (300 MHz, $\left.\mathrm{CDCl}_{3}\right) \delta 3.33-3.27(\mathrm{t}, J=9 \mathrm{~Hz}, 1 \mathrm{H}), 1.90-1.87(\mathrm{~m}, 2 \mathrm{H}), 1.72-1.28(\mathrm{~m}$, 16H) [lit. ${ }^{12}{ }^{1} \mathrm{H}$ NMR $\left.\delta 3.35(1 \mathrm{H}), 1.10-2.50(18 \mathrm{H})\right] ;{ }^{13} \mathrm{C} \mathrm{NMR}\left(75 \mathrm{MHz}, \mathrm{CDCl}_{3}\right) \delta$ 78.2, 44.7, 29.3, 27.4, 25.6, 25.5; MS m/z (rel. intensity, EI, $20 \mathrm{eV}) 168\left(\mathrm{M}^{+}, 1\right), 150$ (2), $99(60), 81$ (100); HRMS (70 eV, $\mathrm{M}^{+}$) calcd. for $\mathrm{C}_{11} \mathrm{H}_{20} \mathrm{O}: 168.1515$, found: 168.1522 . Anal. calcd. for $\mathrm{C}_{11} \mathrm{H}_{20} \mathrm{O}: \mathrm{C}, 78.51 \%, \mathrm{H}, 11.98 \%$. found C, $78.31 \%, \mathrm{H}, 11.92 \%$; IR $\cup 3396 \mathrm{~cm}^{-1}$ (C-OH).

Tris(2-phenylethyl)methanol (22) Compound 22 was prepared according to the preparative procedure for compound 21 in $41 \%$ yield: $\mathrm{mp} 70-72{ }^{\circ} \mathrm{C}$; ${ }^{1} \mathrm{H}$ NMR $\left(300 \mathrm{MHz}, \mathrm{CDCl}_{3}\right) \delta$ 7.33-7.28 (m, $\left.6 \mathrm{H}\right), 7.24-7.17$ $(\mathrm{m}, 9 \mathrm{H}), 2.74-2.68(\mathrm{~m}, 6 \mathrm{H}), 1.93-1.88(\mathrm{~m}, 6 \mathrm{H})$ [lit. ${ }^{15}{ }^{1} \mathrm{H}$ NMR $\delta 7.20(\mathrm{~s}, 15 \mathrm{H}), 2.67(\mathrm{~m}, 6 \mathrm{H}), 1.86(\mathrm{~m}$, $6 \mathrm{H}), 1.33(\mathrm{~s}, 1 \mathrm{H}) ;{ }^{13} \mathrm{C} \mathrm{NMR}\left(75 \mathrm{MHz}, \mathrm{CDCl}_{3}\right.$ ) $\delta 142.2,128.5,128.3,125.9,74.2,41.2,30.1 ; \mathrm{MS} \mathrm{m} / \mathrm{z}$ (rel. 
intensity, EI, $20 \mathrm{eV}$ ) 326 ( $\left.\mathrm{M}^{+}-18,8\right), 239$ (12), 117 (15), 105 (10), 91 (100); HRMS (70 eV, $\mathrm{M}^{+}-18$ ) calcd. for $\mathrm{C}_{25} \mathrm{H}_{26}: 326.2036$, found 326.2031. Anal. calcd. for $\mathrm{C}_{25} \mathrm{H}_{28} \mathrm{O}: \mathrm{C}, 87.16 \%, \mathrm{H}, 8.19 \%$, found $\mathrm{C}, 87.15 \%$, $\mathrm{H}, 8.19 \%$; IR $\cup 3485 \mathrm{~cm}^{-1}$ (C-OH).

Tris(3-phenylpropyl)methanol (23) Compound 23 was prepared according to the preparative procedure for compound 21 in $34 \%$ yield: ${ }^{1} \mathrm{H}$ NMR $\left(300 \mathrm{MHz}, \mathrm{CDCl}_{3}\right) \delta$ 7.35-7.30 (m, 6H), 7.25-7.19 (m, 9H), 2.61 (t. $J=7.3 \mathrm{~Hz}, 6 \mathrm{H}), 1.64-1.57(\mathrm{~m}, 6 \mathrm{H}), 1.55-1.44(\mathrm{~m}, 6 \mathrm{H}) ;{ }^{13} \mathrm{C} \mathrm{NMR}\left(75 \mathrm{MHz}, \mathrm{CDCl}_{3}\right) \delta 142.3,128.4$, 128.3, 125.7, 74.2, 38.6, 36.2, 25.2; MS m/z (rel. intensity, EI, $20 \mathrm{eV}) 368\left(\mathrm{M}^{+}-18,45\right), 291$ (10), 267 (25), 171 (22), 160 (20), 145 (23), 131 (21), 104 (95), 91 (100); HRMS (70 eV, $\mathrm{M}^{+}-18$ ) calcd. for $\mathrm{C}_{28} \mathrm{H}_{32}$ : 368.2496 , found 368.2506 ; IR $\cup 3549 \mathrm{~cm}^{-1}(\mathrm{C}-\mathrm{OH})$.

Tris(3-(benzyloxy)propyl)methanol (24) Compound 24 was prepared according to the preparative procedure for compound 21 in $47 \%$ yield: ${ }^{1} \mathrm{H}$ NMR $\left(300 \mathrm{MHz} \mathrm{CDCl}_{3}\right) \delta$ 7.35-7.24 (m, 15H), $4.51(\mathrm{~s}, 6 \mathrm{H})$, 3.50-3.47 (t. $J=5.9 \mathrm{~Hz}, 6 \mathrm{H}), 1.72-1.64(\mathrm{~m}, 6 \mathrm{H}), 1.58-1.53(\mathrm{~m}, 6 \mathrm{H}) ;{ }^{13} \mathrm{C}$ NMR $\left(75 \mathrm{MHz}, \mathrm{CDCl}_{3}\right) \delta 138.3$, 128.2, 127.5, 127.4, 73.0, 72.8, 70.8, 35.8, 23.9; MS m/z (rel. intensity, EI, $20 \mathrm{eV}) 327\left(\mathrm{M}^{+}+1,10\right), 219$ (15), 129 (95), 111 (42), 106 (13), 91 (100); HRMS (70 eV, M+) calcd. for $\mathrm{C}_{31} \mathrm{H}_{40} \mathrm{O}_{4}: 476.2928$, found 476.2930; IR $v 3445 \mathrm{~cm}^{-1}(\mathrm{C}-\mathrm{OH})$.

Tris(phenylmethyl)methanol (26) Compound 26 was prepared according to the preparative procedure for compound 21 in $53 \%$ yield: $\mathrm{mp} 112-114^{\circ} \mathrm{C}\left[\right.$ lit. $\left.^{16} 110-114{ }^{\circ} \mathrm{C}\right] ;{ }^{1} \mathrm{H} \mathrm{NMR}\left(300 \mathrm{MHz}, \mathrm{CDCl}_{3}\right) \delta 7.40-7.25$ $(\mathrm{m}, 15 \mathrm{H}), 2.83(\mathrm{~s}, 6 \mathrm{H}) ;{ }^{13} \mathrm{C} \mathrm{NMR}\left(75 \mathrm{MHz}, \mathrm{CDCl}_{3}\right) \delta 137.3,130.8,128.1,126.4,73.9,45.8 ; \mathrm{MS} \mathrm{m} / \mathrm{z}$ (rel. intensity, EI, $20 \mathrm{eV}$ ) $284\left(\mathrm{M}^{+}-18,1\right), 211(45), 193(30), 91$ (100); HRMS (70 eV, $\mathrm{M}^{+}-18$ ) calcd. for $\mathrm{C}_{22} \mathrm{H}_{20}$ : 284.1566, found 284.1565 ; IR $v 3569 \mathrm{~cm}^{-1}(\mathrm{OH})$.

(2-Phenyl)thioacetic Acid S-Methyl Ester (27). To a flask with $\mathrm{Ni(acac})_{2}(0.77 \mathrm{~g}, 3.0 \mathrm{mmol})$ was added benzylmagnesium chloride $(1.16 \mathrm{M}, 13 \mathrm{~mL}, 15 \mathrm{mmol})$ at $-78{ }^{\circ} \mathrm{C}$ under argon. The mixture was stirred at $-78{ }^{\circ} \mathrm{C}$ for $1 \mathrm{~h}$, and a solution of DMDTC $(0.61 \mathrm{~g}, 5.0 \mathrm{mmol})$ in THF $(5 \mathrm{~mL})$ was added. The reaction was allowed to proceed at $-78{ }^{\circ} \mathrm{C}$ for $3 \mathrm{~h}$ and quenched by addtion of a saturated $\mathrm{NH}_{4} \mathrm{Cl}$ solution. The crude product was extracted with $\mathrm{CH}_{2} \mathrm{Cl}_{2}$, dried over anhydrous $\mathrm{Na}_{2} \mathrm{SO}_{4}$, concentrated under reduced pressure and purified through liquid chromatography on silica gel, using a solvent gradient from hexanes to $\mathrm{CH}_{2} \mathrm{Cl}_{2} /$ hexanes $=1.5$ as eluent to obtain $27(0.34 \mathrm{~g}, 2.0 \mathrm{mmol}, 40 \%)$ as a slightly yellowish oil: ${ }^{1} \mathrm{H} \mathrm{NMR}\left(300 \mathrm{MHz}, \mathrm{CDCl}_{3}\right) \delta 7.36$ $7.24(\mathrm{~m}, 5 \mathrm{H}), 3.82(\mathrm{~s}, 2 \mathrm{H}), 2.27(\mathrm{~s}, 3 \mathrm{H})$ [lit. ${ }^{17}{ }^{1} \mathrm{H}$ NMR $\left.7.20(\mathrm{~s}, 5 \mathrm{H}), 3.65(\mathrm{~s}, 2 \mathrm{H}), 2.10(\mathrm{~s}, 3 \mathrm{H})\right] ;{ }^{13} \mathrm{C}$ NMR $\left(75 \mathrm{MHz}, \mathrm{CDCl}_{3}\right) \delta 197.7,133.6,129.5,128.6,127.3,50.3,11.8 ; \mathrm{IR} v 1691 \mathrm{~cm}^{-1}(\mathrm{C}=\mathrm{O}$ for thioesters); MS $\mathrm{m} / \mathrm{z}$ (rel. intensity, EI, $20 \mathrm{eV}) 167\left(\mathrm{M}^{+}+1,60\right), 166\left(\mathrm{M}^{+}, 78\right), 139(40), 91(100)$; HRMS (70 eV, $\left.\mathrm{M}^{+}\right)$calcd. for $\mathrm{C}_{9} \mathrm{H}_{10} \mathrm{OS} 166.0453$, found 166.0425 .

(3,3-Dimethyl)thiobutanoic Acid S-Methyl Ester (29) The neopentylmagnesium chloride solution $(0.40 \mathrm{M})$ was prepared from neopentylchloride $(2.5 \mathrm{~g}, 23 \mathrm{mmol})$ and $\mathrm{Mg}(1.5 \mathrm{~g}, 62 \mathrm{mmol})$ in $\mathrm{Et}_{2} \mathrm{O}(15.0 \mathrm{~mL})$ according to the representative procedure. The corresponding cuprate reagent was prepared from $\mathrm{CuI}(1.2 \mathrm{~g}$, $6.0 \mathrm{mmol})$ in $\mathrm{Et}_{2} \mathrm{O}(6 \mathrm{~mL})$ and neopentylmagnesium chloride $(0.4 \mathrm{M}, 30 \mathrm{~mL}, 12 \mathrm{mmol})$ at $-50{ }^{\circ} \mathrm{C}$ for $2 \mathrm{~h}$. To the cuprate solution, a solution of DMDTC $(0.51 \mathrm{~g}, 4.2 \mathrm{mmol})$ in $\mathrm{Et}_{2} \mathrm{O}(4.0 \mathrm{~mL})$ was added. After reaction at $-50{ }^{\circ} \mathrm{C}$ for $0.5 \mathrm{~h}$ and at $\mathrm{rt}$ for $2 \mathrm{~h}$, the mixture was worked up according to the representative procedure to 
provide a crude oil which was purified through liquid chromatography on silica gel, using a eluent gradient from hexanes to $\mathrm{CH}_{2} \mathrm{Cl}_{2} /$ hexanes $=1 / 2$ to obtain $29(0.26 \mathrm{~g}, 1.8 \mathrm{mmol}, 42 \%)$ as a colorless oil: ${ }^{1} \mathrm{H}$ NMR (300 $\left.\mathrm{MHz}, \mathrm{CDCl}_{3}\right) \delta 2.43(\mathrm{~s}, 2 \mathrm{H}), 2.26(\mathrm{~s}, 3 \mathrm{H}), 1.01(\mathrm{~s}, 9 \mathrm{H}) ;{ }^{13} \mathrm{C} \mathrm{NMR}\left(75 \mathrm{MHz}, \mathrm{CDCl}_{3}\right) \delta$ 198.2, 56.6, 31.4, 29.6, 11.7; IR $v 1713 \mathrm{~cm}^{-1}(\mathrm{C}=\mathrm{O})$; $\mathrm{MS} \mathrm{m} / \mathrm{z}$ (rel. intensity, EI, $\left.20 \mathrm{eV}\right) 146\left(\mathrm{M}^{+}, 5\right), 131$ (3), 99 (75), 89 (10), 57 (100); HRMS $\left(\mathrm{M}^{+}\right)$calcd. for $\mathrm{C}_{7} \mathrm{H}_{14} \mathrm{OS}$ : 146.0766 , found 146.0770 .

(2-Trimethylsilyl)thioacetic Acid S-Methyl Ester (32). The trimethylsilylmethylmagnesium chloride solution $(0.97 \mathrm{M})$ was prepared from chloromethyltrimethylsilane $(3.0 \mathrm{~g}, 24 \mathrm{mmol})$ and $\mathrm{Mg}(0.6 \mathrm{~g}, 25 \mathrm{mmol})$ in $\mathrm{Et}_{2} \mathrm{O}$ (24 mL) according to the representative procedure. The corresponding cuprate reagent was prepared from Cul $(1.5 \mathrm{~g}, 7.8 \mathrm{mmol})$ in $\mathrm{Et}_{2} \mathrm{O}(8 \mathrm{~mL})$ and trimethylsilylmethylmagnesium chloride $(16 \mathrm{~mL}, 16 \mathrm{mmol})$ at $-50{ }^{\circ} \mathrm{C}$ for $2 \mathrm{~h}$. To the cuprate solution, a solution of DMDTC $(0.73 \mathrm{~g}, 6.0 \mathrm{mmol})$ in $\mathrm{Et}_{2} \mathrm{O}(6 \mathrm{~mL})$ was added. After reaction at $-50{ }^{\circ} \mathrm{C}$ for $0.5 \mathrm{~h}, 0^{\circ} \mathrm{C}$ for $1.5 \mathrm{~h}$ and at $\mathrm{rt}$ for $2 \mathrm{~h}$, the mixture was worked up according to the representative procedure to provide a crude oil which was purified through liquid chromatography on silica gel, using a solvent gradient from hexanes to $\mathrm{CH}_{2} \mathrm{Cl}_{2} /$ hexanes $=1 / 3$ as eluent to obtain $32(0.67 \mathrm{~g}, 4.1 \mathrm{mmol}, 68$ $\%)$ as a colorless oil: ${ }^{1} \mathrm{H}$ NMR $\left(300 \mathrm{MHz}, \mathrm{CDCl}_{3}\right) \delta 2.30(\mathrm{~s}, 2 \mathrm{H}), 2.24(\mathrm{~s}, 3 \mathrm{H}), 0.10(\mathrm{~s}, 9 \mathrm{H}) ;{ }^{13} \mathrm{C}$ NMR $(75$ $\left.\mathrm{MHz}, \mathrm{CDCl}_{3}\right): \delta 196.9,38.2,11.9,-1.5 ;$ IR $v 1681 \mathrm{~cm}^{-1}(\mathrm{C}=\mathrm{O}) ; \mathrm{MS} \mathrm{m} / \mathrm{z}$ (rel. intensity, EI, $\left.20 \mathrm{eV}\right) 147\left(\mathrm{M}^{+}-\right.$ $15,5), 115(95), 73$ (100); HRMS (70 eV, $\mathrm{M}^{+}-15$ ) calcd. for $\mathrm{C}_{5} \mathrm{H}_{11} \mathrm{OSSi}$ : 147.0294, found 147.0298.

A Representative Procedure for the Formation of Thioesters from DMDTC and Alkylcopper Reagents : Thiononanoic Acid S-Methyl Ester (34). To a slurry of Cul (1.8 g, $9.1 \mathrm{mmol})$ and $\mathrm{PPh}_{3}$ $(2.4 \mathrm{~g}, 9.1 \mathrm{mmol})$ in $\mathrm{Et}_{2} \mathrm{O}(5 \mathrm{~mL})$ was added an ethereal solution of $\mathrm{CH}_{3}\left(\mathrm{CH}_{2}\right)_{7} \mathrm{MgBr}(0.91 \mathrm{M}, 10 \mathrm{~mL})$ at $0{ }^{\circ} \mathrm{C}$ under argon. The mixture was allowed to react at $0{ }^{\circ} \mathrm{C}$ for $40 \mathrm{~min}$, followed by addition of an ethereal solution $(4 \mathrm{~mL})$ of DMDTC $(0.5 \mathrm{~g}, 4.1 \mathrm{mmol})$. The reaction mixture was kept at $0{ }^{\circ} \mathrm{C}$ for $6 \mathrm{~h}$ and quenched by addition of saturated aqueous $\mathrm{NH}_{4} \mathrm{Cl}$ solution. The crude product was extraced with $\mathrm{CH}_{2} \mathrm{Cl}_{2}$ and purified through liquid chromatography on silica gel, using a solvent gradient from hexanes to $\mathrm{CH}_{2} \mathrm{Cl}_{2}$ /hexanes (1/4) as eluent, to provide essentially pure $23(0.3 \mathrm{~g}, 1.6 \mathrm{mmol}, 39 \%)$ as a colorless oil: ${ }^{1} \mathrm{H}$ NMR $\left(300 \mathrm{MHz}, \mathrm{CDCl}_{3}\right) \delta 2.53(\mathrm{l}$, $J=7.5 \mathrm{~Hz}, 2 \mathrm{H}), 2.57(\mathrm{~s}, 3 \mathrm{H}), 1.63(\mathrm{~m}, 2 \mathrm{H}), 1.24(\mathrm{~m}, 10 \mathrm{H}), 0.85(\mathrm{t}, J=6.6 \mathrm{~Hz}, 3 \mathrm{H}) ;{ }^{13} \mathrm{C} \mathrm{NMR}(75 \mathrm{MHz}$, $\left.\mathrm{CDCl}_{3}\right): \delta 200.1,44.0,31.8,29.2,29.1,29.0,25.7,22.6,14.1,11.5 ;$ IR $\cup 1696 \mathrm{~cm}^{-1}(\mathrm{C}=\mathrm{O}$ for thioesters); MS m/z (rel. intensity, EI, $70 \mathrm{eV}) 189\left(\mathrm{M}^{+}+1,72\right), 188\left(\mathrm{M}^{+}, 20\right), 141\left(\mathrm{M}^{+}-\mathrm{SCH}_{3}, 100\right)$; HRMS (70 eV, $\left.\mathrm{M}^{+}\right)$ calcd. for $\mathrm{C}_{10} \mathrm{H}_{20} \mathrm{OS}$ : 188.1236, found 188.1233 .

(4-Phenyl)thiobutanoic Acid S-Methyl Ester (35). Compound 35 was obtained according to the representative procedure as a colorless oil $(35 \%)$ : ${ }^{1} \mathrm{H}$ NMR $\left(300 \mathrm{MHz}, \mathrm{CDCl}_{3}\right) \delta 7.26-7.31(\mathrm{~m}, 2 \mathrm{H}), 7.16-$ $7.22(\mathrm{~m}, 3 \mathrm{H}), 2.65(\mathrm{t}, J=7.5 \mathrm{~Hz}, 2 \mathrm{H}), 2.57(\mathrm{t}, J=7.5 \mathrm{~Hz}, 2 \mathrm{H}), 2.29(\mathrm{~s}, 3 \mathrm{H}), 1.95-2.05(\mathrm{~m}, 2 \mathrm{H}) ;{ }^{13} \mathrm{C} \mathrm{NMR}$ (75 $\mathrm{MHz}, \mathrm{CDCl}_{3}$ ) 199.5, 141.1, 128.4, 128.3, 126.0, 43.1, 34.8, 27.1, 11.5 [lit. ${ }^{18} 199.0,140.9,128.2$, $128.1,125.8,42.8,34.6,26.9,11.2$ ]; IR $v 1688 \mathrm{~cm}^{-1}$ (C=O for thioesters); $\mathrm{MS} \mathrm{m} / \mathrm{z}$ (rel. intensity, EI, $70 \mathrm{eV}$ ) $195\left(\mathrm{M}^{+}+1,20\right), 194\left(\mathrm{M}^{+}, 5\right), 147\left(\mathrm{M}^{+}-\mathrm{SCH}_{3}, 100\right), 91(25)$; HRMS $\left(70 \mathrm{eV}, \mathrm{M}^{+}\right)$calcd. for $\mathrm{C}_{11} \mathrm{H}_{14} \mathrm{OS}$ : 194.0766, found 194.0766.

(Cyclohexane)thiocarboxylic Acid S-Methyl Ester (36) Compound 36 was obtained according to the representative procedures as a colorless oil (37 \%): ${ }^{1} \mathrm{H}$ NMR $\left(300 \mathrm{MHz}, \mathrm{CDCl}_{3}\right) \delta 2.42-2.51(\mathrm{~m}, 1 \mathrm{H})$, $2.24(\mathrm{~s}, 3 \mathrm{H}), 1.89(\mathrm{~d}, J=12.3 \mathrm{~Hz}, 2 \mathrm{H}), 1.78-1.79(\mathrm{~m}, 2 \mathrm{H}), 1,75-1,75(\mathrm{~m}, 1 \mathrm{H}), 1.39-1.50(\mathrm{~m}, 2 \mathrm{H}), 1.15-$ 
$1.35(\mathrm{~m}, 3 \mathrm{H})$ [lit. 17 1.1-2.8 (m, 11H), $2.2(\mathrm{~s}, 3 \mathrm{H})$ ]; $\left.{ }^{13} \mathrm{C} \mathrm{NMR} \mathrm{(75} \mathrm{MHz}, \mathrm{CDCl}_{3}\right)$ 203.5, 52.5, 29.5, 25.6, 25.5, 11.1; IR $v 1692 \mathrm{~cm}^{-1}$ (C=O for thioesters); $\mathrm{MS} \mathrm{m} / \mathrm{z}$ (rel. intensity, $\left.\mathrm{EI}, 70 \mathrm{eV}\right) 158\left(\mathrm{M}^{+}, 6\right), 143\left(\mathrm{M}^{+}\right.$. $\left.\mathrm{CH}_{3}, 10\right), 111\left(\mathrm{M}^{+}-\mathrm{SCH}_{3}, 58\right) ; 83\left(\mathrm{C}_{6} \mathrm{H}_{11}{ }^{+}, 100\right), 55(53)$; HRMS $\left(70 \mathrm{eV}, \mathrm{M}^{+}\right)$calcd. for $\mathrm{C}_{8} \mathrm{H}_{14} \mathrm{OS}$ : 158.0767 , found 158.0770

\section{REFERENCES}

(1) Larock, R. C. Comprehensive Organic Transformations; VCH, New York, 1989.

(2) For examples, see: (a) Cardellicchio, C.; Fiandanese, V.; Marchese, G.; Ronzini, L. Tetrahedron Lett. 1985, 26, 3595; (b) Aoki, S.; Nakamura, E.; Kuwajima, I. Tetrahedron Lett, 1988, 29, 1541; (c) Caniez, G; Normant J. F. Bull. Soc. Chim. Fr. 1977, 570; (d) Seetz, J. W. F. L.; Tol, R. ; Akkerman, O. S.; Bickelhaupt, F. Synthesis 1983, 721; (e) Hlasta, D. J.; Court, J. J. Tetrahedron Lett 1989, 30 , 1773; (f) Whipple, W. L.; Reich, H. J. J. Org. Chem. 1991, 56, 2911.

(3) Leung, M.-k; Lai, J.-L.; Lau, K.-H.; Yu, H.-h.; Hsiao, H. J. J. Org. Chem. 1996, 61, 4175.

(4) For reviews, see: (a) O'Neill, B. T. Comprehensive Organic Synthesis Vol. 1, Trost, B. M.; Fleming, I. Schreiber, S. L. Eds.; Pergamon: Oxford, 1991, 433-435; (b) Bonini, B. F.; Capperuci, ComesFranchini, M.; Degl'Innocenti, A.; Mazzanti, G.; Ricci, A.; Zani, P. Synlett 1993, 937.

(5) Sworin M. and Neumann, W. L. Tetrahedron Lett. 1987, 28, 3217.

(6) (a) Lucast, D. H.; Wemple, J. Tetrahedron Lett. 1977, 1103; (b) Tajima, Y.; Yoshida, A.; Tekeda, N. Oida, S. Tetrahedron Lett. 1985, 26, 673.

(7) Nakamura, E; Hashimoto, K.; Kuwajima, I Bull. Chem. Soc. Jpn.. 1981, 54, 805.

(8) Nakamura, E; Shimizu, M; Kuwajima, I. Tetrahedron Lett. 1976, 1699.

(9) Larson, G. L.; Hernández, Tetrahedron Lett. 1982, 23, 1035.

(10) (a) Saville, M. B.; Shearer, G. J. Chem. Soc. 1925, 592; (b) Tanaka, K.; Matsui, S.; Kaji, A. Bull. Chem. Soc. Jpn. 1980, 53, 3619.

(11) (a) Krapcho, A. P.; Kashdan, D. S.; Jahngen, Jr. E. G. E., J. Org. Chem. 1977, 42, 1189; (b) Devasagayari, A.; Lakshmi, M.; Rao, N.; Periasamy, M. J. Organomet. Chem. 1991, 421, 147.

(12) Funke, C. W.; de Boer, L. M.; Geenevasen, J. A. J.; Cerfontain, H. J. Chem. Soc. Perkin Trans. 2 1976, 1083.

(13) Savoia, D.; Trombini, C.; Umani-Ronchi. A. J. Org. Chem. 1978, 43, 2907.

(14) Hughes, R. J.; Ncube, S.; Pelter, A.; Smith, K. J. Chem. Soc. Perkin Trans. 1 1977, 1172.

(15) Komatsu, K. ; Shirai, S.; Tomioka, I.; Okamoto, K. Bull. Chem. Soc. Jpn. 1984, 57, 1377.

(16) Ruggli, P.; Hegudus, B. Helv. Chim. Acta 1942, 25, 1285.

(17) Seebach, D.; Burstinghaus, R. Synthesis 1975, 461.

(18) Otten, P. A.; Oskam, N.; van der Gen, A. Tetrahedron 1996, 33, 11095. 